\title{
New general Grüss-type inequalities over $\sigma$-finite measure space with applications
}

\author{
Sajid lqbal' ${ }^{1}$, Muhammad Adil Khan², Thabet Abdeljawad ${ }^{3,45^{*}}$ (D, Muhammad Samraiz ${ }^{6}$, \\ Gauhar Rahman ${ }^{7}$ and Kottakkaran Sooppy Nisar ${ }^{8}$
}

\section{"Correspondence:}

tabdeljawad@psu.edu.sa

${ }^{3}$ Department of Mathematics and

General Sciences, Prince Sultan

University, Riyadh, 12345, Saudi

Arabia

${ }^{4}$ Department of Medical Research, China Medical University, Taichung, 40402, Taiwan

Full list of author information is available at the end of the article

\begin{abstract}
In this paper, we establish some new integral inequalities involving general kernels. We obtain the related broad range of fractional integral inequalities. Also, we apply the Young inequality to find new forms of inequalities for generalized kernels. These new and motivated results generalize the results for fractional integrals such that fractional integral of a function with respect to an increasing function, Riemann-Lioville fractional integrals, Erdélyi-Kober fractional integrals, Hadamard fractional integrals, generalized factional integral integrals in addition to the corresponding $k$-fractional integrals.
\end{abstract}

MSC: 26D15; 26D10; 26A33; 34B27

Keywords: Grüss-type inequalities; Kernel; Fractional integrals; Young's inequality

\section{Introduction}

Fractional calculus deals with the study of derivative and integral operators of fractional order. This field is as important as calculus itself. In the last few decades, it attracted many researchers, who produced remarkable work (see, e.g., [1-3, 6, 7, 9-13, 18]). In particular, the uniqueness of solutions for fractional partial differential equations can be established by using fractional integral inequalities.

The Grüss inequality connects the integral of the product of two functions with the product of their integrals. Our main purpose in this paper is showing some new modifications of the Grüss inequality by using a general kernel. The Grüss inequality is one of the most fascinating inequalities and stated in the following theorem.

Theorem 1.1 ([5]) Let $\Re$ be a set of real numbers, let $m, M, n, N \in \Re$, and let $\Omega, \Upsilon$ : $\left[\tau_{1}, \tau_{2}\right] \rightarrow \Re$ be two positive functions such that $m \leq \Omega(\mu) \leq M$ and $n \leq \Upsilon(\mu) \leq N$ for $\mu \in\left[\tau_{1}, \tau_{2}\right]$. Then

$$
\begin{aligned}
& \mid \frac{1}{\tau_{2}-\tau_{1}} \int_{\tau_{1}}^{\tau_{2}} \Omega(\mu) \Upsilon(\mu) d \mu \\
& \quad-\frac{1}{\left(\tau_{2}-\tau_{1}\right)^{2}} \int_{\tau_{1}}^{\tau_{2}} \Omega(\mu) d \mu \int_{\tau_{1}}^{\tau_{2}} \Upsilon(\mu) d \mu \mid
\end{aligned}
$$

(c) The Author(s) 2020. This article is licensed under a Creative Commons Attribution 4.0 International License, which permits use, sharing, adaptation, distribution and reproduction in any medium or format, as long as you give appropriate credit to the original author(s) and the source, provide a link to the Creative Commons licence, and indicate if changes were made. The images or other third party material in this article are included in the article's Creative Commons licence, unless indicated otherwise in a credit line to the material. If material is not included in the article's Creative Commons licence and your intended use is not permitted by statutory regulation or exceeds the permitted use, you will need to obtain permission directly from the copyright holder. To view a copy of this licence, visit http://creativecommons.org/licenses/by/4.0/. 


$$
\leq \frac{1}{4}(M-m)(N-n)
$$

where the constant $\frac{1}{4}$ cannot be improved.

Let $(\Delta, \Sigma, \mu)$ be a measure space with positive $\sigma$-finite measure, let $k: \Delta \times \Delta \rightarrow \mathbb{R}$ be a nonnegative function, and let

$$
\Theta(x)=\int_{\Delta} k(x, y) d \mu(y), \quad x \in \Delta .
$$

Throughout this paper, we suppose $\Theta(x)>0$ a.e. on $\Delta$.

Let $U(k)$ denote the class of functions $\Lambda: \Delta \rightarrow \mathbb{R}$ with the representation

$$
\bar{\Lambda}(x)=\int_{\Delta} k(x, y) \Lambda(y) d \mu(y),
$$

where $\bar{\Lambda}: \Delta \rightarrow \mathbb{R}$ is a measurable function.

Definition 1.2 Let $f \in L_{1}([a, b])$ (the Lebesgue measure). The left-sided and right-sided Riemann-Liouville fractional integrals $I_{a^{+}}^{\alpha} f$ and $I_{b^{-}}^{\alpha} f$ of order $\alpha>0$ are defined by

$$
I_{a^{+}}^{\alpha} f(x)=\frac{1}{\Gamma(\alpha)} \int_{a}^{x} f(y)(x-y)^{\alpha-1} d y \quad(x>a)
$$

and

$$
I_{b^{-}}^{\alpha} f(x)=\frac{1}{\Gamma(\alpha)} \int_{x}^{b} f(y)(y-x)^{\alpha-1} d y \quad(x<b)
$$

where $\Gamma$ is the gamma function.

Diaz et al. [4] defined the gamma $k$-function as follows.

Definition 1.3 The function $\Gamma_{k}$, a generalization of the classical gamma function, is defined as follows:

$$
\Gamma_{k}(t)=\lim _{n \rightarrow \infty} \frac{n ! k^{n}(n k)^{\frac{t}{k}-1}}{(t)_{n, k}}, \quad k>0, \mathfrak{R}(t)>0,
$$

where $(t)_{n, k}=t(t+k)(t+2 k) \ldots,(t+(n-1) k), n \geq 1$, is the Pochhammer $k$ symbol. Its integral representation is given by

$$
\Gamma_{k}(t)=\int_{0}^{\infty} x^{t-1} e^{\frac{-x^{k}}{k}} d x, \quad \mathfrak{R}(t)>0 .
$$

In particular, for $k=1, \Gamma_{1}(t)=\Gamma(t)$.

Definition 1.4 ([12]) Let $f \in L_{1}([a, b])$ (the Lebesgue measure). The left-sided and rightsided Riemann-Liouville fractional integrals $I_{a^{+}}^{\alpha, k} f$ and $I_{b^{-}}^{\alpha, k} f$ of order $\alpha, k>0$ are defined 
by

$$
I_{a^{+}}^{\alpha, k} f(x)=\frac{1}{k \Gamma_{k}(\alpha)} \int_{a}^{x} f(y)(x-y)^{\frac{\alpha}{k}-1} d y \quad(x>a)
$$

and

$$
I_{b^{-}}^{\alpha, k} f(x)=\frac{1}{k \Gamma_{k}(\alpha)} \int_{x}^{b} f(y)(y-x)^{\frac{\alpha}{k}-1} d y \quad(x<b),
$$

where $\Gamma_{k}(\alpha)$ is the $k$-gamma function.

Definition 1.5 ([10]) Let $(a, b),-\infty \leq a<b \leq \infty$, be a finite or infinite interval of the real line $\Re$, and let $\alpha>0$. Let $g$ be an increasing and positive monotone function on $(a, b]$. The left- and right-sided fractional integrals of a function $f$ with respect to $g$ in $[a, b]$ are given by

$$
I_{a+; g}^{\alpha} f(x)=\frac{1}{\Gamma(\alpha)} \int_{a}^{x} \frac{g^{\prime}(t) f(t) d t}{[g(x)-g(t)]^{1-\alpha}}, \quad x>a,
$$

and

$$
I_{b-; j}^{\alpha} f(x)=\frac{1}{\Gamma(\alpha)} \int_{x}^{b} \frac{g^{\prime}(t) f(t) d t}{[g(t)-g(x)]^{1-\alpha}}, \quad x<b .
$$

A more general form of Definition 1.5 is as follows.

Definition 1.6 Let $k>0$, let $(a, b),-\infty \leq a<b \leq \infty$, be a finite or infinite interval of the real line $\Re$, and let $\alpha>0$. Let $g$ be an increasing and positive monotone function on $(a, b]$. The left- and right-sided fractional integrals of a function $f$ with respect to $g$ of order $\alpha, k>0$ in $[a, b]$ are given by

$$
I_{a+; g}^{\alpha, k} f(x)=\frac{1}{\Gamma_{k}(\alpha)} \int_{a}^{x} \frac{g^{\prime}(t) f(t) d t}{[g(x)-g(t)]^{1-\frac{\alpha}{k}}}, \quad x>a,
$$

and

$$
I_{b-; g}^{\alpha, k} f(x)=\frac{1}{\Gamma(\alpha)} \int_{x}^{b} \frac{g^{\prime}(t) f(t) d t}{[g(t)-g(x)]^{1-\frac{\alpha}{k}}}, \quad x<b .
$$

Now we continue with the definition of Hadamard-type fractional integrals.

Definition 1.7 Let $(a, b)$ be a finite or infinite interval of the half-axis $\Re_{+}$, and and $\alpha>0$. The left-sided and right-sided Hadamard-type fractional integrals of order $\alpha>0$ are given by

$$
J_{a_{+}}^{\alpha} f(x)=\frac{1}{\Gamma(\alpha)} \int_{a}^{x}\left(\log \frac{x}{y}\right)^{\alpha-1} \frac{f(y) d y}{y}, \quad x>a,
$$


and

$$
J_{b}^{\alpha} f(x)=\frac{1}{\Gamma(\alpha)} \int_{x}^{b}\left(\log \frac{y}{x}\right)^{\alpha-1} \frac{f(y) d y}{y}, \quad x<b,
$$

respectively.

The generalized Hadamard-type fractional integrals are defined as follows.

Definition 1.8 Let $(a, b)$ be a finite or infinite interval of $\mathbb{R}^{+}$, and let $\alpha>0$. The left- and right-sided Hadamard-type fractional integrals of order $\alpha>0$ are given by

$$
J_{a_{+}}^{\alpha} f(x)=\frac{1}{\Gamma_{k}(\alpha)} \int_{a}^{x}\left(\log \frac{x}{y}\right)^{\frac{\alpha}{k}-1} \frac{f(y) d y}{y}, \quad x>a,
$$

and

$$
J_{b}^{\alpha} f(x)=\frac{1}{\Gamma_{k}(\alpha)} \int_{x}^{b}\left(\log \frac{y}{x}\right)^{\frac{\alpha}{k}-1} \frac{f(y) d y}{y}, \quad x<b,
$$

respectively.

Note that Hadamard fractional integrals of order $\alpha$ are a particular case of the left- and right-sided fractional integrals of a function $f$ with respect to the function $g(x)=\log (x)$ in $[a, b]$, where $0 \leq a<b \leq \infty$.

Now we present the definition of the Erdélyi-Kober-type fractional integrals. Some of these definitions and results were presented in Samko et al. [16].

Definition 1.9 Let $(a, b)(0 \leq a<b \leq \infty)$ be a finite or infinite interval of the half-axis $\mathbb{R}^{+}$. Let $\alpha>0, \sigma>0$, and $\eta \in \mathbb{R}$. We consider the left- and right-sided integrals of order $\alpha \in \mathbb{R}$ defined by

$$
I_{a_{+} ; \sigma ; \eta}^{\alpha} f(x)=\frac{\sigma x^{-\sigma(\alpha+\eta)}}{\Gamma(\alpha)} \int_{a}^{x} \frac{t^{\sigma \eta+\sigma-1} f(t) d t}{\left(x^{\sigma}-t^{\sigma}\right)^{1-\alpha}}
$$

and

$$
I_{b_{-} ; \sigma ; \eta}^{\alpha} f(x)=\frac{\sigma x^{\sigma \eta}}{\Gamma(\alpha)} \int_{x}^{b} \frac{t^{\sigma(1-\eta-\alpha)-1} f(t) d t}{\left(t^{\sigma}-x^{\sigma}\right)^{1-\alpha}}
$$

respectively. Integrals (1.4) and (1.5) are called the Erdélyi-Kober-type fractional integrals.

\section{Main results}

The first main result is given in the following:

Theorem 2.1 Let $(\Delta, \Sigma, \mu)$ be a measure space with positive $\sigma$-finite measure, let $k$ : $\Delta \times \Delta \rightarrow \mathbb{R}$ be a nonnegative function, and let $\Omega \in U(k)$ be a positive function on $[0, \infty)$. Suppose that the exist integrable functions $\Psi_{1}, \Psi_{2}$ on $[0, \infty)$, such that

$$
\Psi_{1}(\xi) \leq \Omega(\xi) \leq \Psi_{2}(\xi)
$$


for all $\xi \in[0, \infty)$. Then

$$
\bar{\Psi}_{1}(\xi) \bar{\Omega}(\xi)+\bar{\Psi}_{2}(\xi) \bar{\Omega}(\xi) \geq \bar{\Psi}_{1}(\xi) \bar{\Psi}_{2}(\xi)+\bar{\Omega}(\xi) \bar{\Omega}(\xi)
$$

Proof Using (2.1), for all $\gamma \geq 0$ and $\delta \geq 0$, we have

$$
\left[\Psi_{2}(\gamma)-\Omega(\gamma)\right]\left[\Omega(\delta)-\left(\Psi_{1}\right)(\delta)\right] \geq 0
$$

Then

$$
\Psi_{2}(\gamma) \Omega(\delta)+\Psi_{1}(\delta) \Omega(\gamma) \geq \Psi_{1}(\delta) \Psi_{2}(\gamma)+\Omega(\gamma) \Omega(\delta) .
$$

Multiplying both sides of (2.3) by $k(\xi, \gamma)$ and integrating the resulting identity with respect to $\gamma$ over $\Delta$, we get

$$
\begin{aligned}
& \Omega(\delta) \int_{\Delta} k(\xi, \gamma) \Psi_{2}(\gamma) d \mu(\gamma)+\Psi_{1}(\delta) \int_{\Delta} k(\xi, \gamma) \Omega(\gamma) d \mu(\gamma) \\
& \quad \geq \Psi_{1}(\delta) \int_{\Delta} k(\xi, \gamma) \Psi_{2}(\gamma) d \mu(\gamma)+\Omega(\delta) \int_{\Delta} k(\xi, \gamma) \Omega(\gamma) d \mu(\gamma),
\end{aligned}
$$

which can be written as

$$
\Omega(\delta) \bar{\Psi}_{2}(\xi)+\Psi_{1}(\delta) \bar{\Omega}(\xi) \geq \Psi_{1}(\delta) \bar{\Psi}_{2}(\xi)+\Omega(\delta) \bar{\Omega}(\xi) .
$$

Now multiplying both sides of $(2.4)$ by $k(\xi, \delta)$ and integrating the resulting identity with respect to $\delta$ over $\Delta$, we get

$$
\bar{\Psi}_{1}(\xi) \bar{\Omega}(\xi)+\bar{\Psi}_{2}(\xi) \bar{\Omega}(\xi) \geq \bar{\Psi}_{1}(\xi) \bar{\Psi}_{2}(\xi)+\bar{\Omega}(\xi) \bar{\Omega}(\xi) .
$$

This completes the proof.

Corollary 2.2 Let $m, M \in \Re$, with $m<M$, and let $\xi>0$. Let $\Omega$ be a positive function such that $m \leq \Omega(\xi) \leq M$. Then

$$
m \Theta(\xi) \bar{\Omega}(\xi)+M \Theta(\xi) \bar{\Omega}(\xi) \geq m M \Theta(\xi) \Theta(\xi)+\bar{\Omega}(\xi) \bar{\Omega}(\xi)
$$

where

$$
\Theta(\xi)=\int_{\Delta} k(\xi, \gamma) d \mu_{2}(\gamma), \quad \xi \in \Delta
$$

Remark 2.3 Applying Theorem 2.1 and Corollary 2.2 with $\Delta=(a, b), d \mu(\gamma)=d \gamma$, and $d \mu(\delta)=d \delta$, we have

$$
k(x, y)= \begin{cases}\frac{g^{\prime}(y)}{k \Gamma_{k}(\alpha)(g(x)-g(y))^{1-\frac{\alpha}{k}},} & a \leq y \leq x, \\ 0, & x<y \leq b .\end{cases}
$$


We get that $\Theta(x)=\frac{1}{\Gamma_{k}(\alpha+k)}(g(x)-g(a))^{\frac{\alpha}{k}}$. Substituting $\bar{\Psi}_{1}=I_{a+; g}^{\alpha, k} \Psi_{1}, \bar{\Psi}_{2}=I_{a+;}^{\alpha, k} \Psi_{2}$, and $\bar{\Omega}=$ $I_{a+g}^{\alpha, k} \Omega$, we get Theorem 2.1 and Corollary 2.3 of [15], respectively. In particular, taking $k=1$, we get Theorem 2.11 and Corollary 2.14 of [8], respectively.

Remark 2.4 Applying Theorem 2.1 and Corollary 2.2 with $\Delta=(a, b), d \mu(\gamma)=d \gamma$, and $d \mu(\delta)=d \delta$, we have

$$
k(x, y)= \begin{cases}\frac{1}{k \Gamma_{k}(\alpha)(x-y)^{1-\frac{\alpha}{k}},} & a \leq y \leq x, \\ 0, & x<y \leq b .\end{cases}
$$

We get that $\Theta(x)=\frac{1}{\Gamma_{k}(\alpha+k)}(x-a)^{\frac{\alpha}{k}}$. Substituting $\bar{\Psi}_{1}=I_{a^{+}}^{\alpha, k} \Psi_{1}, \bar{\Psi}_{2}=I_{a^{+}}^{\alpha, k} \Psi_{2}$, and $\bar{\Omega}=I_{a^{+}}^{\alpha, k} \Omega$ in Corollary 2.2, we get Corollary 2.2 of [15], respectively. Moreover, taking $k=1$, we get Theorem 2 and Corollary 3 of [17], respectively.

Remark 2.5 Applying Theorem 2.1 and Corollary 2.2 with $\Delta=(a, b), d \mu(\gamma)=d \gamma$, and $d \mu(\delta)=d \delta$, we have

$$
k(x, y)= \begin{cases}\frac{1}{y k \Gamma_{k}(\alpha)(\log x-\log y)^{1-\frac{\alpha}{k}}}, & a \leq y \leq x, \\ 0, & x<y \leq b .\end{cases}
$$

We get that $\Theta(\xi)=\frac{1}{\Gamma_{k}(\alpha+k)}(\log \xi-\log a)^{\frac{\alpha}{k}}$. Substituting $\bar{\Psi}_{1}=J_{a_{+}}^{\alpha} \Psi_{1}, \bar{\Psi}_{2}=J_{a_{+}}^{\alpha} \Psi_{2}$, and $\bar{\Omega}=$ $J_{a_{+}}^{\alpha} \Omega$, we get the results for the Hadamard-type fractional integrals.

Remark 2.6 Applying Theorem 2.1 and Corollary 2.2 with $\Delta=(a, b), d \mu(\gamma)=d \gamma$, and $d \mu(\delta)=d \delta$, we have

$$
k(x, y)= \begin{cases}\frac{1}{\Gamma(\alpha)} \frac{\sigma x^{-\sigma(\alpha+\eta)}}{\left(x^{\sigma}-y^{\sigma}\right)^{1-\alpha}} y^{\sigma \eta+\sigma-1}, & a \leq y \leq x \\ 0, & x<y \leq b\end{cases}
$$

We get that $\Theta(x)=\frac{1}{\Gamma(\alpha+1)}\left(1-\left(\frac{a}{x}\right)^{\sigma}\right)_{2}^{\alpha} F_{1}\left(\alpha,-\eta ; \alpha+1 ; 1-\left(\frac{a}{x}\right)^{\sigma}\right)$. Substituting $\bar{\Psi}_{1}=I_{a_{+} ; \sigma ; \eta}^{\alpha} \Psi_{1}$, $\bar{\Psi}_{2}=I_{a_{+} ; \sigma ; \eta}^{\alpha} \Psi_{2}$, and $\bar{\Omega}=I_{a_{+} ; \sigma ; \eta}^{\alpha} \Omega$, we get the results for Erdélyi-Kober fractional integral.

Theorem 2.7 Let $(\Delta, \Sigma, \mu)$ be a measure space with positive $\sigma$-finite measure, let $k$ : $\Delta \times \Delta \rightarrow \mathbb{R}$ be a non-egative function, let $\Omega, \Psi_{1}, \Psi_{2}, \varphi_{1}, \varphi_{2}, \Upsilon \in U(k)$, and let $\Omega$ and $\Upsilon$ be positive functions on $[0, \xi)$. Suppose that (2.1) holds and there exist integrable functions $\varphi_{1}$ and $\varphi_{2}$ on $[0, \xi)$ such that

$$
\varphi_{1}(\xi) \leq \Upsilon(\xi) \leq \varphi_{1}(\xi)
$$

Then the following inequalities hold:
(a) $\bar{\varphi}_{1}(\xi) \bar{\Omega}(\xi)+\bar{\Psi}_{2}(\xi) \bar{\Upsilon}(\xi) \geq \bar{\varphi}_{2}(\xi) \bar{\Psi}_{2}(\xi)+\bar{\Omega}(\xi) \bar{\Upsilon}(\xi)$,
(b) $\bar{\Psi}_{1}(\xi) \bar{\Upsilon}(\xi)+\bar{\varphi}_{2}(\xi) \bar{\Omega}(\xi) \geq \bar{\Psi}_{1}(\xi) \bar{\varphi}_{2}(\xi)+\bar{\Omega}(\xi) \bar{\Upsilon}(\xi)$,
(c) $\bar{\Psi}_{2}(\xi) \varphi_{2}(\xi)+\bar{\Omega}(\xi) \bar{\Upsilon}(\xi) \geq \bar{\Psi}_{2}(\xi) \bar{\Upsilon}(\xi)_{2}(\xi)+\bar{\Omega}(\xi) \bar{\varphi}(\xi)$,
(d) $\quad \bar{\Psi}_{1}(\xi) \bar{\varphi}_{1}(\xi)+\bar{\Omega}(\xi) \bar{\Upsilon}(\xi) \geq \bar{\Psi}_{1}(\xi) \bar{\Upsilon}(\xi)+\bar{\varphi}_{1}(\xi) \bar{\Omega}(\xi)$. 
Proof For all $\xi \in[0, \infty)$, from (2.1) and (2.10) it follows that

$$
\left.\left.\left[\Psi_{2}(\gamma)-\Omega(\gamma)\right)\right]\left[(\Upsilon)(\delta)-\varphi_{1}(\delta)\right)\right] \geq 0
$$

Then

$$
\Psi_{2}(\gamma)(\Upsilon)(\delta)+\varphi_{1}(\delta) \Omega(\gamma) \geq \varphi_{1}(\delta) \Psi_{2}(\gamma)+\Omega(\gamma) \Upsilon(\delta)
$$

Multiplying both sides by $k(\xi, \gamma)$ and integrating the resulting identity with respect to $\gamma$ over the interval $\Delta$, we have that

$$
\begin{aligned}
& \Upsilon(\delta) \int_{\Delta} k(\xi, \gamma) \Psi_{2}(\gamma) d \mu(\gamma)+\varphi_{1}(\delta) \int_{\Delta} k(\xi, \gamma) \Omega(\gamma) d \mu(\gamma) \\
& \quad \geq \varphi_{1}(\delta) \int_{\Delta} k(\xi, \gamma) \Psi_{2}(\gamma) d \mu(\gamma)+\Upsilon(\delta) \int_{\Delta} k(\xi, \gamma) \Omega(\gamma) d \mu(\gamma),
\end{aligned}
$$

which can be written as

$$
\Upsilon(\delta) \bar{\Psi}_{2}(\xi)+\varphi_{1}(\delta) \bar{\Omega}(\xi) \geq \varphi_{1}(\delta) \bar{\Psi}_{2}(\xi)+\Upsilon(\delta) \bar{\Omega}(\xi)
$$

Again multiplying both sides by $k(\xi, \delta)$ and integrating the resulting identity with respect to $\delta$ over $\Delta$, we have that

$$
\bar{\varphi}_{1}(\xi) \bar{\Omega}(\xi)+\bar{\Psi}_{2}(\xi) \bar{\Upsilon}(\xi) \geq \bar{\varphi}_{1}(\xi) \bar{\Psi}_{2}(\xi)+\bar{\Omega}(\xi) \bar{\Upsilon}(\xi)
$$

This completes the proof of part (a).

To prove parts (b)-(d), we will use the following inequalities:

$$
\begin{aligned}
& \left(\varphi_{2}(\gamma)-\Upsilon(\gamma)\right)\left(\Omega(\delta)-\Psi_{1}(\delta)\right) \geq 0 \\
& \left(\Psi_{2}(\gamma)-\Omega(\gamma)\right)\left(\Upsilon(\delta)-\varphi_{2}(\delta)\right) \geq 0 \\
& \left(\Psi_{1}(\gamma)-\Omega(\gamma)\right)\left(\Upsilon(\delta)-\varphi_{1}(\delta)\right) \geq 0 .
\end{aligned}
$$

Corollary 2.8 Let the assumptions of Theorem 2.7 be satisfied. Suppose that there exist real constants $m, M, n, N$ such that $m \leq \Omega(\xi) \leq M$ and $n \leq \Upsilon(\xi) \leq N$ for all $\xi \in[0, \infty)$. Then
(a) $n \Theta(\xi) \bar{\Omega}(\xi)+M \Theta(\xi) \bar{\Upsilon}(\xi) \geq n M \Theta(\xi) \Theta(\xi)+\bar{\Omega}(\xi) \bar{\Upsilon}(\xi)$
(b) $m \Theta(\xi) \Upsilon(\xi)+N \Theta(\xi) \Omega(\xi) \geq m N \Theta(\xi) m \Theta(\xi)+\bar{\Omega}(\xi) \bar{\Upsilon}(\xi)$,
(c) $N M \Theta(\xi) \Theta(\xi)+\bar{\Omega}(\xi) \bar{\Upsilon}(\xi) \geq M \Theta(\xi) \bar{\Upsilon}(\xi)+N \Theta(\xi) \bar{\Omega}(\xi)$,
(d) $n m \Theta(\xi) \Theta(\xi) \bar{\varphi}_{1}(\xi)+\bar{\Omega}(\xi) \bar{\Upsilon}(\xi) \geq m \Theta(\xi) \bar{\Upsilon}(\xi)+n m \Theta(\xi) \bar{\Omega}(\xi)$,

where $\Theta(\xi)$ is defined by $(2.5)$.

Remark 2.9 Choosing the kernel $k(x, y)$ defined by (2.6) and substituting $\bar{\Psi}_{1}=I_{a+g}^{\alpha, k} \Psi_{1}$, $\bar{\Psi}_{2}=I_{a+; g}^{\alpha, k} \Psi_{2}$, and $\bar{\Omega}=I_{a+g}^{\alpha, k} \Omega$ in Theorem 2.7 and Corollary 2.8, we get Theorem 2.5 and 
Corollary 2.6 of [15], respectively. In particular, taking $k=1$, we get Theorem 2.15 and Corollary 2.16 of [8], respectively.

Remark 2.10 Choosing the kernel $k(x, y)$ defined by (2.7) and substituting $\bar{\Psi}_{1}=I_{a^{+}}^{\alpha, k} \Psi_{1}$, $\bar{\Psi}_{2}=I_{a^{+}}^{\alpha, k} \Psi_{2}$, and $\bar{\Omega}=I_{a^{+}}^{\alpha, k} \Omega$ in Theorem 2.7 and Corollary 2.8, we get Theorem 2.5 and Corollary 2.6 of [15], respectively. In particular, by taking $k=1$ Theorem 2.7 leads to Theorem 2.15 of [17].

Remark 2.11 Choosing the kernel $k(x, y)$ defined by $(2.8)$ and $\Theta(\xi)=\frac{1}{\Gamma_{k}(\alpha+k)}(\log \xi-\log a)^{\frac{\alpha}{k}}$ and substituting $\bar{\Psi}_{1}=J_{a_{+}}^{\alpha} \Psi_{1}, \bar{\Psi}_{2}=J_{a_{+}}^{\alpha} \Psi_{2}$, and $\bar{\Omega}=J_{a_{+}}^{\alpha} \Omega$ in Theorem 2.7 and Corollary 2.8, we get the inequalities for Haadmard-type fractional integrals.

Remark 2.12 Choosing the kernel $k(x, y)$ defined by $(2.9)$ and

$$
\Theta(\xi)=\frac{1}{\Gamma(\alpha+1)}\left(1-\left(\frac{a}{x}\right)^{\sigma}\right)_{2}^{\alpha} F_{1}\left(\alpha,-\eta ; \alpha+1 ; 1-\left(\frac{a}{x}\right)^{\sigma}\right)
$$

and substituting $\bar{\Psi}_{1}=\left(I_{a_{+} ; \sigma ; \eta}^{\alpha} \Psi_{1}\right), \bar{\Psi}_{2}=\left(I_{a_{+} ; \sigma ; \eta}^{\alpha} \Psi_{2}\right)$, and $\bar{\Omega}=\left(I_{a_{+} ; \sigma ; \eta}^{\alpha} \Omega\right)$ in Theorem 2.7 and Corollary 2.8, we get the result for the Erdélyi-Kober fractional integral.

Lemma 2.13 Let $(\Delta, \Sigma, \gamma)$ be a measure space with positive $\sigma$-finite measure, let $k: \Delta \times$ $\Delta \rightarrow \mathbb{R}$ be a nonnegative function, and let $\Omega, \Psi_{1}, \Psi_{2} \in U(k)$ be such that $\Omega$ is positive on $[0, \infty)$ and $\Psi_{1}, \Psi_{2}$ are integrable on $[0, \infty)$. Then

$$
\begin{aligned}
\Theta(\xi) & \overline{\Omega^{2}}(\xi)-(\bar{\Omega}(\xi))^{2} \\
= & \left(\bar{\Psi}_{2}(\xi)-\bar{\Omega}(\xi)\right)\left(\bar{\Omega}(\xi)-\bar{\Psi}_{1}(\xi)\right)-\Theta(\xi)\left(\bar{\Psi}_{2}(\xi)-\bar{\Omega}(\xi)\right)\left(\bar{\Omega}(\xi)-\bar{\Psi}_{1}(\xi)\right) \\
& +\Theta(\xi) \overline{\Psi_{1}(\xi) \Omega(\xi)}-\bar{\Psi}_{1}(\xi) \bar{\Omega}(\xi)+\Theta(\xi) \overline{\Psi_{2}(\xi) \Omega(\xi)}-\bar{\Psi}_{2}(\xi) \bar{\Omega}(\xi) \\
& -\Theta(\xi) \overline{\Psi_{1}(\xi) \Psi_{2}(\xi)}+\bar{\Psi}_{1}(\xi) \bar{\Psi}_{2}(\xi)
\end{aligned}
$$

where $\Theta(\xi)$ is defined by (2.5).

Proof Since $\gamma, \delta>0$, we have

$$
\begin{aligned}
\left(\Psi_{2}(\delta)\right. & -\Omega(\delta))\left(\Omega(\gamma)-\Psi_{1}(\gamma)\right)+\left(\Psi_{2}(\gamma)-\Omega(\gamma)\right)\left(\Omega(\delta)-\Psi_{1}(\delta)\right) \\
& -\left(\Psi_{2}(\gamma)-\Omega(\gamma)\right)\left(\Omega(\delta)-\Psi_{1}(\delta)\right)-\left(\Psi_{2}(\delta)-\Omega(\delta)\right)\left(\Omega(\delta)-\Psi_{1}(\delta)\right) \\
= & \Omega^{2}(\gamma)+(\Omega(\delta))^{2}-2 \Omega(\gamma) \Omega(\delta)+\Psi_{2}(\delta) \Omega(\gamma)+\Psi_{1}(\gamma) \Omega(\delta) \\
& -\Psi_{1}(\gamma) \Psi_{2}(\delta)+\Psi_{2}(\delta) \Omega(\delta)+\Psi_{1}(\delta) \Omega(\gamma)-\Psi_{1}(\delta) \Psi_{2}(\gamma) \\
& -\Psi_{2}(\delta) \Omega(\gamma)+\Psi_{1}(\gamma) \Psi_{2}(\gamma)-\Psi_{1}(\gamma) \Omega(\gamma)-\Psi_{2}(\delta) \Omega(\delta) \\
& +\Psi_{1}(\delta) \Psi_{2}(\delta)-\Psi_{1}(\delta) \Omega(\delta) .
\end{aligned}
$$

Multiplying both sides by $k(\xi, \gamma)$ and integrating with respect to the variable $\gamma$ over $\Delta$, we get

$$
\left(\Psi_{2}(\delta)-\Omega(\delta)\right)\left(\bar{\Omega}(\xi)-\bar{\Psi}_{1}(\xi)\right)+\left(\bar{\Psi}_{2}(\xi)-\bar{\Omega}(\xi)\right)\left(\Omega(\delta)-\Psi_{1}(\delta)\right)
$$




$$
\begin{aligned}
& -\overline{\left(\Psi_{2}(\gamma)-\Omega(\gamma)\right)\left(\Omega(\delta)-\Psi_{1}(\delta)\right)}-\left(\Psi_{2}(\delta)-\Omega(\delta)\right)\left(\Omega(\delta)-\Psi_{1}(\delta)\right) \Theta(\xi) \\
& =\overline{\Omega^{2}}(\gamma)+\Theta(\xi) \Omega^{2}(\delta)-2 \bar{\Omega}(\xi) \Omega(\delta)+\Psi_{2}(\delta) \bar{\Omega}(\xi)+\bar{\Psi}_{1}(\xi) \Omega(\delta) \\
& -\bar{\Psi}_{1}(\xi) \Psi_{2}(\delta)+\bar{\Psi}_{2}(\xi) \Omega(\delta)+\Psi_{1}(\delta) \bar{\Omega}(\xi)-\Psi_{1}(\delta) \bar{\Psi}_{2}(\xi) \\
& -\overline{\Psi_{2}(\xi) \Omega(\xi)}+\overline{\Psi_{1}(\xi) \Psi_{2}(\xi)} \Theta(\xi)-\overline{\Psi_{1}(\xi) \Omega(\xi)}-\Psi_{2}(\delta) \Omega(\delta) \Theta(\xi) \\
& +\Theta(\xi) \Psi_{1}(\delta) \Psi_{2}(\delta)-\Psi_{1}(\delta) \Omega(\delta) \Theta(\xi) .
\end{aligned}
$$

Again multiplying both sides by $k(\xi, \delta)$ and integrating with respect to the variable $\delta$ over $\Delta$, we get

$$
\begin{aligned}
& \left(\bar{\Psi}_{2}(\xi)-\bar{\Omega}(\xi)\right)\left(\bar{\Omega}_{(\xi)}-\bar{\Psi}_{1}(\xi)\right)+\left(\bar{\Psi}_{2}(\xi)-\bar{\Omega}(\xi)\right)\left(\bar{\Omega}_{(\xi)}-\bar{\Psi}_{1}(\xi)\right) \\
& -\Theta(\xi) \overline{\left(\Psi_{2}(\xi)-\Omega(\xi)\right)\left(\Omega(\xi)-\Psi_{1}(\xi)\right)}-\left(\bar{\Psi}_{2}(\xi)-\bar{\Omega}(\xi)\right)\left(\bar{\Omega}(\xi)-\bar{\Psi}_{1}(\xi)\right) \Theta(\xi) \\
& =\overline{\Omega^{2}}(\xi) \Theta(\xi)+\Theta(\xi) \Omega^{2}(\xi)-2 \bar{\Omega}(\xi) \bar{\Omega}(\xi)+\bar{\Psi}_{2}(\xi) \bar{\Omega}(\xi)+\bar{\Psi}_{1}(\gamma) \bar{\Omega}(\xi) \\
& -\bar{\Psi}_{1}(\xi) \bar{\Psi}_{2}(\xi)+\bar{\Psi}_{2}(\xi) \bar{\Omega}(\xi)+\bar{\Psi}_{1}(\xi) \bar{\Omega}(\xi)-\bar{\Psi}_{1}(\xi) \bar{\Psi}_{2}(\xi) \\
& -\Theta(\xi) \overline{\Psi_{2}(\xi) \Omega(\xi)}+\overline{\Psi_{1}(\xi) \Psi_{2}(\xi)} \Theta(\xi)-\Theta(\xi) \overline{\Psi_{1}(\xi) \Omega(\xi)}-\overline{\Psi_{2}(\xi) \Omega(\xi)} \Theta(\xi) \\
& +\Theta(\xi) \overline{\Psi_{1}(\xi) \Psi_{2}(\xi)}-\overline{\Psi_{1}(\xi) \Omega(\xi)} \Theta(\xi) \text {. }
\end{aligned}
$$

This completes the proof.

Corollary 2.14 Let $m<M, k>0$, and let $\Omega$ be a positive function on $[0, \xi)$ such that $m \leq$ $\Omega(\xi) \leq M$. Then

$$
\begin{aligned}
& \Theta(\xi)\left(\overline{\Omega^{2}}(\xi)\right)-(\bar{\Omega}(\xi))^{2} \\
& \quad=(M \Theta(\xi)-\bar{\Omega}(\xi))(\bar{\Omega}(\xi)-m \Theta(\xi))-\Theta(\xi)((\overline{(M-\Omega(\xi))(\Omega(\xi)-m)})
\end{aligned}
$$

where $\Theta(\xi)$ is defined by (2.5).

Remark 2.15 Taking the kernel $k(x, y)$ defined by (2.6) and substituting $\bar{\Psi}_{1}=I_{a+; g}^{\alpha, k} \Psi_{1}$, $\bar{\Psi}_{2}=I_{a+; g}^{\alpha, k} \Psi_{2}, \bar{\Omega}=I_{a+; g}^{\alpha, k} \Omega, \overline{\Psi_{1} \Omega}=I_{a+; g}^{\alpha, k} \Psi_{1} \Omega, \overline{\Psi_{2} \Omega}=I_{a+; g}^{\alpha, k} \Psi_{2} \Omega, \overline{\Psi_{1} \Psi_{2}}=I_{a+; g}^{\alpha, k} \Psi_{1} \Psi_{2}$, and $\overline{\left(\Psi_{2}(\xi)-\Omega(\xi)\right)\left(\Omega(\xi)-\Psi_{1}(\xi)\right)}=I_{a+; g}^{\alpha, k}\left(\Psi_{2}(\xi)-\Omega(\xi)\right)\left(\Omega(\xi)-\Psi_{1}(\xi)\right)$ in Theorem 2.13 and Corollary 2.14, we get Lemma 2.9 and Corollary 2.11 of [15], respectively. In particular, by taking $k=1$ we get Theorem 2.19 and Corollary 2.11 of [8], respectively.

Remark 2.16 Taking the kernel $k(x, y)$ defined by (2.7) and substituting $\bar{\Psi}_{1}=I_{a^{+}}^{\alpha, k} \Psi_{1}$, $\bar{\Psi}_{2}=I_{a^{+}}^{\alpha, k} \Psi_{2}, \bar{\Omega}=I_{a^{+}}^{\alpha, k} \Omega, \overline{\Psi_{1} \Omega}=I_{a^{+}}^{\alpha, k} \Psi_{1} \Omega, \overline{\Psi_{2} \Omega}=I_{a^{+}}^{\alpha, k} \Psi_{2} \Omega, \overline{\Psi_{1} \Psi_{2}}=I_{a^{+}}^{\alpha, k} \Psi_{1} \Psi_{2}$, and $\overline{\left(\Psi_{2}(\xi)-\Omega(\xi)\right)\left(\Omega(\xi)-\Psi_{1}(\xi)\right)}=I_{a^{+}}^{\alpha, k}\left(\Psi_{2}(\xi)-\Omega(\xi)\right)\left(\Omega(\xi)-\Psi_{1}(\xi)\right)$ in Lemma 2.13, we get Corollary 2.10 of [15]. In particular, by taking $k=1$ Lemma 2.13 and Corollary 2.14 become Lemma 7 and Corollary 8 of [17], respectively.

Remark 2.17 Taking the kernel $k(x, y)$ defined by (2.8) and substituting $\Theta(\xi)=\frac{1}{\Gamma_{k}(\alpha+k)} \times$ $(\log \xi-\log a)^{\frac{\alpha}{k}}, \bar{\Psi}_{1}=J_{a_{+}}^{\alpha} \Psi_{1}, \bar{\Psi}_{2}=J_{a_{+}}^{\alpha} \Psi_{2}, \bar{\Omega}=J_{a_{+}}^{\alpha} \Omega$, and $\overline{\left(\Psi_{2}(\xi)-\Omega(\xi)\right)\left(\Omega(\xi)-\Psi_{1}(\xi)\right)}=$ $J_{a_{+}}^{\alpha}\left(\Psi_{2}(\xi)-\Omega(\xi)\right)\left(\Omega(\xi)-\Psi_{1}(\xi)\right)$ in Theorem 2.7 and Corollary 2.8, we get the inequalities for Hadamard fractional integrals. 
Remark 2.18 Taking the kernel $k(x, y)$ defined by (2.9) and substituting $\Theta(x)=$ $\frac{1}{\Gamma(\alpha+1)}\left(1-\left(\frac{a}{x}\right)^{\sigma}\right)_{2}^{\alpha} F_{1}\left(\alpha,-\eta ; \alpha+1 ; 1-\left(\frac{a}{x}\right)^{\sigma}\right), \bar{\Psi}_{1}=I_{a_{+} ; \sigma ; \eta}^{\alpha} \Psi_{1}, \bar{\Psi}_{2}=I_{a_{+} ; \sigma ; \eta}^{\alpha} \Psi_{2}, \bar{\Omega}=I_{a_{+} ; \sigma ; \eta}^{\alpha} \Omega$, and $\frac{\bar{T}\left(\Psi_{2}(\xi)-\Omega(\xi)\right)\left(\Omega(\xi)-\Psi_{1}(\xi)\right)}{=} I_{a_{+} ; \sigma ; \eta}^{\alpha}\left(\Psi_{2}(\xi)-\Omega(\xi)\right)\left(\Omega(\xi)-\Psi_{1}(\xi)\right)$ in Theorem 2.7 and Corollary 2.8, we get the result for the Erdélyi-Kober fractional integral.

Theorem 2.19 Let $(\Delta, \Sigma, \gamma)$ be a measure spaces with positive $\sigma$-finite measure, let $k$ : $\Delta \times \Delta \rightarrow \mathbb{R}$ be a nonnegative function, and let $\Omega, \Psi_{1}, \Psi_{2}, \varphi_{1}, \varphi_{2}, \Upsilon \in U(k)$ be integrable functions on $[0, \xi)$. If conditions (2.1) and (2.10) are satisfied, then

$$
|\Theta(\xi) \overline{\Omega(\xi) \Upsilon(\xi)}-\bar{\Omega}(\xi) \bar{\Upsilon}(\xi)| \leq \sqrt{T\left(\Omega, \Psi_{1}, \Psi_{2}\right) T\left(\Upsilon, \varphi_{1}, \varphi_{2}\right)}
$$

where

$$
\begin{aligned}
T\left(\Omega, \Psi_{1}, \Psi_{2}\right)= & \left(\bar{\Psi}_{2}(\xi)-\bar{\Omega}(\xi)\right)\left(\bar{\Omega}(\xi)-\bar{\Psi}_{1}(\xi)\right)+\Theta(\xi) \overline{\Psi_{1}(\xi) \Omega(\xi)}-\bar{\Psi}_{1}(\xi) \bar{\Omega}(\xi) \\
& +\Theta(\xi) \overline{\Psi_{2}(\xi) \Omega(\xi)}-\bar{\Psi}_{2}(\xi) \bar{\Omega}(\xi)+\bar{\Psi}_{1}(\xi) \bar{\Psi}_{2}(\xi)-\Theta(\xi) \overline{\Psi_{1}(\xi) \Psi_{2}(\xi)}
\end{aligned}
$$

and

$$
\begin{aligned}
T\left(\Upsilon, \varphi_{1}, \varphi_{2}\right)= & \left(\bar{\varphi}_{2}(\xi)-\bar{\Upsilon}(\xi)\right)\left(\bar{\Upsilon}(\xi)-\bar{\varphi}_{1}(\xi)\right)+\Theta(\xi) \bar{\varphi}_{1}(\xi) \bar{\Upsilon}(\xi)-\bar{\varphi}_{1}(\xi) \bar{\Upsilon}(\xi) \\
& +\Theta(\xi) \overline{\varphi_{2}(\xi) \Upsilon(\xi)}-\bar{\varphi}_{2}(\xi) \bar{\Upsilon}(\xi)+\bar{\varphi}_{1}(\xi) \bar{\varphi}_{2}(\xi)-\Theta(\xi) \overline{\varphi_{1}(\xi) \varphi_{2}(\xi)}
\end{aligned}
$$

with $\Theta(\xi)$ defined by (2.5).

Proof Let $\xi>0, \gamma, \delta \in[0, \xi]$, let $\Omega, \Upsilon$ be two positive functions on $[0, \infty)$ such that conditions (2.1) and (2.10) are satisfied, and let $T(\gamma, \delta)$ be defined by

$$
T(\gamma, \delta)=(\Omega(\gamma)-\Omega(\delta))(\Upsilon(\gamma)-\Upsilon(\delta)) .
$$

Multiplying both sides (2.12) by $\frac{1}{2} k(\xi, \gamma) k(\xi, \delta)$ and integrating with respect to the variables $\gamma$ and $\delta$ over $\Delta$ and $\Delta$, we get

$$
\frac{1}{2} \int_{\Delta} \int_{\Delta} k(\xi, \delta) k(\xi, \gamma) T(\gamma, \delta) d \mu(\gamma) d \mu(\delta)=\Theta(\xi) \overline{\Omega(\xi) \Upsilon(\xi)}-\bar{\Omega}(\xi) \bar{\Upsilon}(\xi) .
$$

Applying the Cauchy-Schwarz inequality, we get

$$
\begin{aligned}
& \left(\frac{1}{2} \int_{\Delta} \int_{\Delta} k(\xi, \gamma) k(\xi, \delta)(\Omega(\gamma)-\Omega(\delta))(\Upsilon(\gamma)-\Upsilon(\delta)) d \mu(\gamma) d \mu(\delta)\right)^{2} \\
& \leq \int_{\Delta} \int_{\Delta} k(\xi, \gamma) k(\xi, \delta)(\Omega(\gamma)-\Omega(\delta))^{2} d \mu(\gamma) d \mu(\delta) \\
& \quad \times \int_{\Delta} \int_{\Delta} k(\xi, \gamma) k(\xi, \delta)(\Upsilon(\gamma)-\Upsilon(\delta))^{2} d \mu(\gamma) d \mu(\delta) .
\end{aligned}
$$

From (2.13) and (2.14) we get

$$
(\Theta(\xi) \overline{\Omega(\xi) \Upsilon(\xi)}-\Omega(\xi) \Upsilon(\xi))^{2} \leq\left(\Theta(\xi) \overline{\Omega^{2}}(\xi)-(\bar{\Omega}(\xi))^{2}\right)\left(\Theta(\xi) \overline{\Upsilon^{2}}(\xi)-\bar{\Upsilon}(\xi)^{2}\right) .
$$


Since $\left(\Psi_{2}(\xi)-\Omega(\xi)\right)\left(\Omega(\xi)-\Psi_{1}(\xi)\right) \geq 0$ and $\left(\varphi_{2}(\xi)-\Upsilon(\xi)\right)\left(\Upsilon(\xi)-\varphi_{1}(\xi)\right) \geq 0$, we have

$$
\Theta(\xi) \overline{\left(\Psi_{2}(\xi)-\Omega(\xi)\right)\left(\Omega(\xi)-\Psi_{1}(\xi)\right)} \geq 0
$$

and

$$
\Theta(\xi) \overline{\left(\varphi_{2}(\xi)-\Upsilon(\xi)\right)\left(\Upsilon(\xi)-\varphi_{1}(\xi)\right)} \geq 0
$$

Thus from Lemma 2.13 we have

$$
\begin{aligned}
\Theta(\xi) & \overline{\Omega^{2}}(\xi)-(\bar{\Omega}(\xi))^{2} \\
\leq & \left(\bar{\Psi}_{2}(\xi)-\bar{\Omega}(\xi)\right)\left(\bar{\Omega}(\xi)-\bar{\Psi}_{1}(\xi)\right)+\Theta(\xi) \overline{\Psi_{1}(\xi) \Omega(\xi)} \bar{\Psi}_{1}(\xi) \bar{\Omega}(\xi) \\
& +\Theta(\xi) \overline{\Psi_{2}(\xi) \Omega(\xi)}-\bar{\Psi}_{2}(\xi) \bar{\Omega}(\xi)+\bar{\Psi}_{1}(\xi) \bar{\Psi}_{2}(\xi)-\Theta(\xi) \overline{\Psi_{1}(\xi) \Psi_{2}(\xi)} \\
& =T\left(\Omega, \Psi_{1}, \Psi_{2}\right)
\end{aligned}
$$

and

$$
\begin{aligned}
\Theta(\xi) & \overline{\Upsilon^{2}}(\xi)-(\bar{\Upsilon}(\xi))^{2} \\
\leq & \left(\bar{\varphi}_{2}(\xi)-\bar{\Upsilon}(\xi)\right)\left(\bar{\Upsilon}(\xi)-\bar{\varphi}_{1}(\xi)\right)+\Theta(\xi) \overline{\varphi_{1}(\xi) \Upsilon(\xi)}-\bar{\varphi}_{1}(\xi) \bar{\Upsilon}(\xi) \\
& +\Theta(\xi) \overline{\varphi_{2}(\xi) \Upsilon(\xi)}-\bar{\varphi}_{2}(\xi) \bar{\Upsilon}(\xi)+\bar{\varphi}_{1}(\xi) \bar{\varphi}_{2}(\xi)-\Theta(\xi) \overline{\varphi_{1}(\xi) \varphi_{2}(\xi)} \\
= & T\left(\Upsilon, \varphi_{1}, \varphi_{2}\right) .
\end{aligned}
$$

Therefore inequality (2.11) follows from (2.15) and (2.16). This completes the proof.

Corollary 2.20 Let $m, M, n, N \in \Re, T\left(\Omega, \Psi_{1}, \Psi_{2}\right)=T(\Omega, m, M)$, and $T\left(\Upsilon, \varphi_{1}, \varphi_{2}\right)=$ $T(\Upsilon, n, N)$. Then inequality (2.11) reduces to

$$
|\Theta(\xi) \overline{\Omega(\xi) \Upsilon(\xi)}-\bar{\Omega}(\xi) \bar{\Upsilon}(\xi)| \leq(\Theta(\xi))^{2}(M-m)(N-n)
$$

Remark 2.21 Taking the kernel $k(x, y)$ defined by (2.6) and substituting $\bar{\Omega}=I_{a+; g}^{\alpha, k} \Omega, \bar{\Upsilon}=$ $I_{a+; g}^{\alpha, k} \Upsilon$, and $\overline{\Omega \Upsilon}=I_{a+; g}^{\alpha, k} \Omega \Upsilon$ in Theorem 2.19 and Corollary 2.20, we get Theorem 2.13 and Corollary 2.14 of [15], respectively. In particular, choosing $k=1$, Theorem 2.13 and and Corollary 2.14 lead to Theorem 2.23 and Corollary 2.26 of [8], respectively.

Remark 2.22 Taking the kernel $k(x, y)$ defined by (2.7) and substituting $\bar{\Omega}_{1}=I_{a^{+}}^{\alpha, k} \Omega, \bar{\Upsilon}=$ $I_{a^{+}}^{\alpha, k} \Upsilon$, and $\overline{\Omega \Upsilon}=I_{a^{+}}^{\alpha, k} \Omega \Upsilon$ in Theorem 2.19 and Corollary 2.20, we get the results for the Riemann-Liouville integral. In particular, taking $k=1$, Theorem 2.19 gives Theorem 9 of [17], and Corollary 2.20 gives Remark 10 of [17].

Remark 2.23 Taking the kernel $k(x, y)$ defined by (2.8) and substituting $\Theta(\xi)=\frac{1}{\Gamma_{k}(\alpha+k)} \times$ $(\log \xi-\log a)^{\frac{\alpha}{k}}, \bar{\Omega}=J_{a_{+}}^{\alpha} \Omega, \bar{\Upsilon}=J_{a_{+}}^{\alpha} \Psi_{2}$, and $\overline{\Omega \Upsilon}=J_{a_{+}}^{\alpha} \Omega \Upsilon$ in Theorem 2.19 and Corollary 2.20, we get the inequalities for Hadamard fractional integrals. 
Remark 2.24 Taking the kernel $k(x, y)$ defined by (2.9) and substituting $\Theta(x)=\frac{1}{\Gamma(\alpha+1)}(1-$ $\left.\left(\frac{a}{x}\right)^{\sigma}\right)_{2}^{\alpha} F_{1}\left(\alpha,-\eta ; \alpha+1 ; 1-\left(\frac{a}{x}\right)^{\sigma}\right), \bar{\Psi}_{1}=\left(I_{a_{+} ; \sigma ; \eta}^{\alpha} \Psi_{1}\right) \bar{\Psi}_{2}=\left(I_{a_{+} ; \sigma ; \eta}^{\alpha} \Psi_{2}\right)$, and $\bar{\Omega}=\left(I_{a_{+} ; \sigma ; \eta}^{\alpha} \Omega\right)$ in Theorem 2.19 and Corollary 2.20, we get the result for the Erdélyi-Kober-type fractional integral.

Theorem 2.25 Let $k>0$, and let $\Omega$ and $\Upsilon$ be positive functions defined on $[0, \infty)$. Then the following inequalities hold:

1. $q \overline{\Omega^{p}}(\xi)+p \overline{\Upsilon q}(\xi) \geq p q \frac{1}{\Theta(\xi)} \bar{\Upsilon}(\xi) \bar{\Omega}(\xi)$,

2. $q \overline{\Omega^{p}}(\xi) \overline{\Upsilon^{p}}(\xi)+p \overline{\Omega^{q}}(\xi) \overline{\Upsilon^{q}}(\xi) \geq p q(\overline{\Omega(\xi) \Upsilon(\xi)})^{2}$,

3. $q \overline{\Omega^{p}}(\xi) \overline{\Upsilon^{p}}(\xi)+p \overline{\Omega^{q}}(\xi) \overline{\Upsilon^{q}}(\xi) \geq p q \overline{\Omega(\xi) \Upsilon^{p-1}(\xi) \Omega(\xi) \Upsilon^{q-1}(\xi)}$,

4. $q \overline{\Omega^{p}}(\xi) \overline{\Upsilon^{q}}(\xi)+p \overline{\Omega^{p}}(\xi) \overline{\Upsilon^{q}}(\xi) \geq p q \overline{\Omega^{p-1}(\xi) \Upsilon^{q-1}(\xi) \Omega(\xi) \Upsilon(\xi)}$,

5. $q \overline{\Omega^{p}}(\xi) \overline{\Upsilon^{2}}(\xi)+p \overline{\Omega^{2}}(\xi) \overline{\Upsilon^{q}}(\xi) \geq p q \overline{\Omega(\xi) \Upsilon(\xi)} \overline{\Omega^{\frac{2}{q}}(\xi) \Upsilon^{\frac{2}{p}}(\xi)}$,

6. $q \overline{\Omega^{2}}(\xi) \overline{\Upsilon^{q}}(\xi)+p \overline{\Omega^{p}}(\xi) \overline{\Upsilon^{2}}(\xi) \geq p q \overline{\Omega^{\frac{2}{p}}(\xi) \Upsilon^{\frac{2}{q}}(\xi)} \overline{\Omega^{p-1}(\xi) \Upsilon^{q-1}(\xi)}$,

7. $q \overline{\Omega^{2}(\xi) \Upsilon^{q}(\xi)}+p \overline{\Omega^{2}(\xi) \Upsilon^{p}}(\xi) \geq p q \frac{1}{\Theta(\xi)} \overline{\Omega^{\frac{2}{p}}(\xi) \Upsilon^{q-1}(\xi) \Omega^{\frac{2}{q}}(\xi) \Upsilon^{p-1}(\xi)}$,

where $\Theta(\xi)$ is defined by (2.5).

Proof By Young's inequality we have

$$
\frac{a^{p}}{p}+\frac{a^{q}}{q} \geq a b\left(a, b \geq 0, p, q>1, \frac{1}{p}+\frac{1}{q}=1\right) .
$$

Taking $a=\Omega(\gamma)$ and $b=\Upsilon(\delta)$, we have

$$
\frac{(\Omega(\gamma))^{p}}{p}+\frac{(\Upsilon(\delta))^{q}}{q} \geq \Omega(\gamma) \Upsilon(\delta)
$$

for all $\Omega(\gamma), \Upsilon(\delta) \geq 0$.

Multiplying by $k(\xi, \gamma)$ and integrating with respect to $\gamma$ over the interval $\Delta$, we get

$$
\int_{\Delta} k(\xi, \gamma) \frac{\Omega^{p}(\gamma)}{p} d \mu(\gamma)+\int_{\Delta} k(\xi, \gamma) \frac{\Upsilon^{q}(\delta)}{q} d \mu(\gamma) \geq \int_{\Delta} k(\xi, \gamma) \Omega(\gamma) \Upsilon(\delta) d \mu(\gamma),
$$

which becomes

$$
\frac{1}{p} \overline{\Omega^{p}}(\xi)+\frac{1}{q} \Upsilon^{q}(\delta) \Theta(\xi) \geq \Upsilon(\delta) \bar{\Omega}(\xi) .
$$

Again multiplying by $k(\xi, \delta)$ and integrating with respect to the variable $\delta$ over the interval $\Delta$, we get

$$
\frac{1}{p} \overline{\Omega^{p}}(\xi) \Theta(\xi)+\frac{1}{q} \overline{\Upsilon q}(\xi) \Theta(\xi) \geq \bar{\Upsilon}(\xi) \bar{\Omega}(\xi),
$$


which implies that

$$
\frac{1}{p} \overline{\Omega^{p}}(\xi)+\frac{1}{q} \overline{\Upsilon q}(\delta) \geq \frac{1}{\Theta(\xi)} \bar{\Upsilon}(\xi) \bar{\Omega}(\xi) .
$$

This completes the proof of part (a).

The remaining inequalities can be proved using Young's inequality in a similar manner by taking:

2. $a=\Omega(\gamma)(\Upsilon)(\delta)$ and $b=\Omega(\delta) \Upsilon(\gamma)$,

3. $a=\frac{\Omega(\gamma)}{\Upsilon(\gamma)} \quad$ and $\quad b=\frac{\Omega(\delta)}{\Upsilon(\delta)}, \quad \Upsilon(\gamma) \Upsilon(\delta) \neq 0$,

4. $a=\frac{\Omega(\delta)}{\Omega(\gamma)} \quad$ and $\quad b=\frac{\Upsilon(\delta)}{\Upsilon(\gamma)}, \quad \Omega(\gamma) \Upsilon(\delta) \neq 0$,

5. $a=\Omega(\gamma) \Upsilon^{\frac{2}{p}}(\delta)$ and $b=\Omega^{\frac{2}{q}}(\delta) \Upsilon(\gamma)$,

6. $a=\frac{\Omega^{\frac{2}{p}}(\gamma)}{\Omega(\delta)} \quad$ and $\quad b=\frac{\Upsilon^{\frac{2}{q}}(\gamma)}{\Upsilon(\gamma)}, \quad \Omega(\delta) \Upsilon(\delta) \neq 0$,

7. $a=\frac{\Omega^{\frac{2}{p}}(\gamma)}{\Upsilon(\delta)} \quad$ and $\quad b=\frac{\Omega^{\frac{2}{q}}(\delta)}{\Upsilon(\gamma)}, \quad \Upsilon(\gamma) \Upsilon(\delta) \neq 0$.

Example 2.26 Let $k>0$, and let $\varphi_{2}(\gamma)$ be a positive function on $[0, \infty)$, and let $m=$ $\min _{0 \leq \gamma \leq \xi} \frac{\Omega(\gamma)}{\gamma(\gamma)}$ and $M=\max _{0 \leq \gamma \leq \xi} \frac{\Omega(\gamma)}{\gamma(\gamma)}$. Then we have

$$
0 \leq \overline{\Omega^{2}}(\xi) \overline{\Upsilon^{2}}(\xi) \leq \frac{(m+M)^{2}}{4 m M}(\overline{\Omega(\xi) \Upsilon(\xi)})^{2}
$$

Proof From the min and max conditions we have that

$$
\left(\frac{\Omega(\gamma)}{\Upsilon(\gamma)}-m\right)\left(M-\frac{\Omega(\gamma)}{\Upsilon(\gamma)}\right) \Upsilon^{2}(\gamma) \geq 0
$$

and

$$
\Omega^{2}(\gamma)+m M \Upsilon^{2}(\gamma) \leq(m+M) \Omega(\gamma) \Upsilon(\gamma) .
$$

Multiplying by $k(\xi, \gamma)$ and integrating with respect to the variable $\gamma$ over the interval $\Delta$, we get

$$
\begin{gathered}
\int_{\Delta} k(\xi, \gamma) \Omega^{2}(\gamma) d \mu(\gamma)+m M \int_{\Delta} k(\xi, \gamma) \Upsilon^{2}(\gamma) d \mu(\gamma) \\
\leq(m+M) \int_{\Delta} k(\xi, \gamma) \Omega(\gamma) \Upsilon(\gamma) d \mu(\gamma) .
\end{gathered}
$$

This implies that

$$
\overline{\Omega^{2}}(\xi)+m M \overline{\Upsilon^{2}}(\xi) \leq(m+M) \overline{\Omega(\xi)(\Upsilon)(\xi)} .
$$


Alternatively, it follows from

$$
\left(\sqrt{\overline{\Omega^{2}}(\xi)}-\sqrt{m M \overline{\Upsilon^{2}}(\xi)}\right)^{2} \geq 0
$$

that

$$
2 \sqrt{\overline{\Omega^{2}}(\xi)} \sqrt{m M \overline{\Upsilon^{2}}(\xi)} \leq \overline{\Omega^{2}}(\gamma)+m M \overline{\Upsilon^{2}}(\gamma) .
$$

Therefore from (2.18) and (2.19) it follows that

$$
4 m M \overline{\Omega^{2}}(\xi) \overline{\Upsilon^{2}}(\xi) \leq(m+M)^{2}(\overline{\Omega(\xi)(\Upsilon)(\xi)})^{2},
$$

and the proof is complete.

Remark 2.27 Applications for the discussed fractional integrals can be given, but we omit the details.

\section{Concluding remarks}

Recently, Rahman [14], Rashid [15], and Kacar [8] studied a broad range of Grüss-type inequalities for different kinds of fractional integrals. Although papers [14, 15], and [8] are connected in the sense that one generalizes another, we observe that there may be a great generalization that covers all possible kinds of fractional integrals mentioned in these papers. Therefore we introduced a special class of transformations that involve general kernels over $\sigma$-finite measure and prove all the results. Motivated by the above, we successfully presented certain elegant inequalities, which generalize all the previous results.

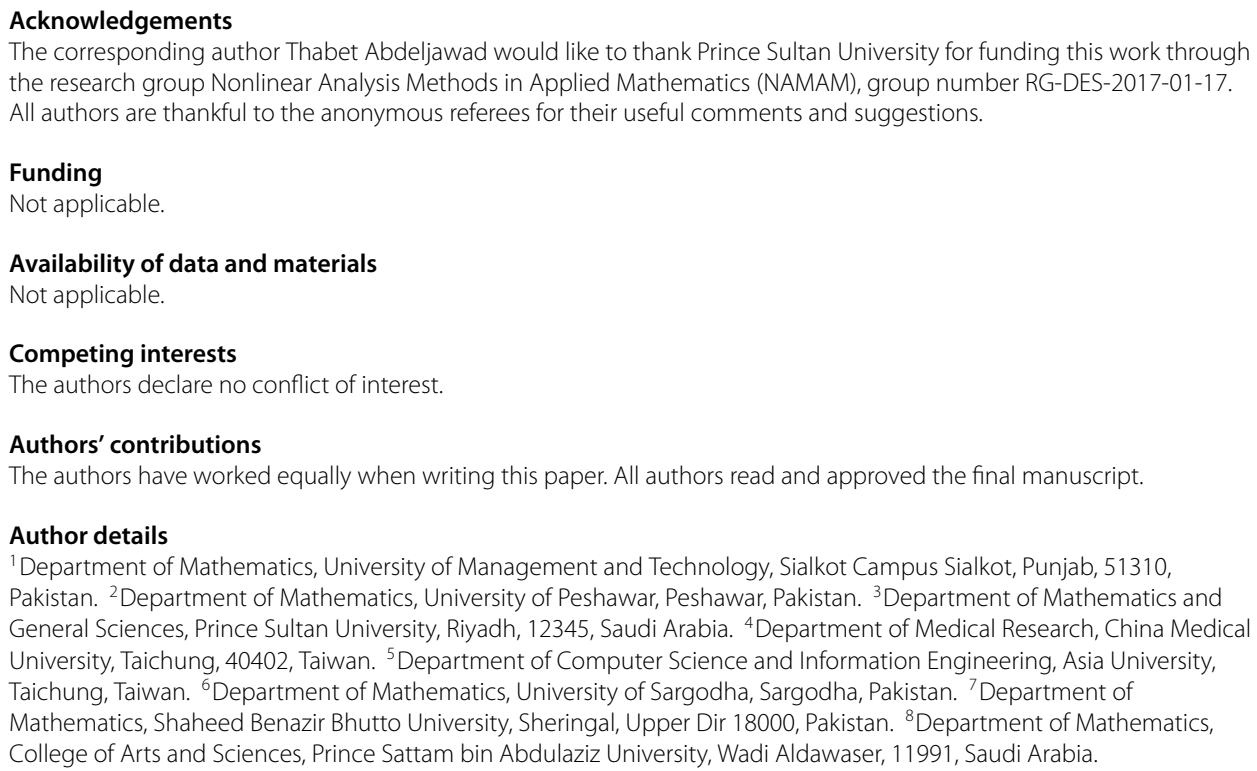

\section{Publisher's Note}

Springer Nature remains neutral with regard to jurisdictional claims in published maps and institutional affiliations. 
References

1. Akman, T., Yildiz, B., Baleanu, D.: New discretization of Caputo-Fabrizio derivative. Comput. Appl. Math. 37(3), 3307-3333 (2018)

2. Anastassiou, G.A.: Fractional Differentiation Inequalities. Springer, Berlin (2009)

3. Baleanu, D., Diethelm, K., Scalas, E., Trujillo, J.J.: Fractional Calculus: Models and Numerical Methods. World Scientific, Hackensack (2012)

4. Diaz, R., Pariguan, E.: On hypergeometric functions and Pochhammer k-symbol. Divulg. Mat. 15, 179-192 (2007)

5. Grüss, G.: Uber das Maximum des absoluten Betrages $\frac{1}{b-a} \int_{a}^{b} f(x) g(x) d x-\frac{1}{(b-a)^{2}} \int_{a}^{b} f(x) d x \int_{a}^{b} g(x) d x$. Math. Z. 39(1), 215-226 (1935)

6. Hilfer, R.: Applications of Fractional Calculus in Physics. World Scientific, Singapore (2000)

7. Jarad, F., Abdeljawad, T., Baleanu, B.: On the generalized fractional derivatives and their Caputo modification. J. Nonlinear Sci. Appl. 10(5), 2607-2619 (2017)

8. Kacar, E., Kacar, Z., Yildirim, H.: Integral inequalities for Riemann-Liouville fractional integrals of a function with respect to another function. Iran. J. Math. Sci. Inform. 13(1), 1-13 (2018)

9. Katugampola, U.N.: New approach to generalized fractional integral. Appl. Math. Comput. 218, 860-865 (2011)

10. Kilbas, A.A., Srivastava, H.M., Trujillo, J.J.: Theory and Applications of Fractional Differential Equations. North-Holland Mathematics Studies, vol. 204. Elsevier, New York (2006)

11. Mainardi, F.: Fractional Calculus and Waves in Linear Viscoelasticity. Imperial College Press, London (2010)

12. Mubeen, S., Habibullah, G.M.: k-Fractional integrals and application. Int. J. Contemp. Math. Sci. 7(2), 89-94 (2012)

13. Oliveira, D.S., de Oliveira, E.C.: Hilfer-Katugampola fractional derivatives. Comput. Appl. Math. 37, 3672-3690 (2018). https://doi.org/10.1007/s40314-017-0536-8

14. Rahman, G., Nisar, K.S., Abdeljawad, T.: Certain Hadamard proportional fractional integral inequalities. Mathematics 8 , 504 (2020)

15. Rashid, S., Jarad, F., Noor, M.A., Noor, K.I., Baleanu, D., Liu, J.: On Grüss inequalities within generalized $k$-fractional integrals. Adv. Differ. Equ. 2020, 203 (2020)

16. Samko, S.G., Kilbas, A.A., Marichev, O.I.: Fractional Integrals and Derivatives: Theory and Applications. Gordon \& Breach, New York (1993)

17. Tariboon, J., Ntouyas, S.K., Sudsutad, W.: Some new Riemann-Liouville fractional integral inequalities. Int. J. Math. Math. Sci. 2014, Article ID 869434 (2014)

18. Tomovski, Z., Hilfer, R., Srivastava, H.M.: Fractional and operational calculus with generalized fractional derivative operators and Mittag-Leffler type functions. Integral Transforms Spec. Funct. 21(11), 797-814 (2010)

\section{Submit your manuscript to a SpringerOpen ${ }^{\circ}$ journal and benefit from:}

- Convenient online submission

- Rigorous peer review

- Open access: articles freely available online

- High visibility within the field

- Retaining the copyright to your article

Submit your next manuscript at $\gg$ springeropen.com 